\title{
DETERMINACIÓN DE RESIDUOS DE OXITETRACICLINA EN MUESTRAS DE CARNE BOVINA
}

\author{
Sandra Milena Acosta Agudelo ${ }^{1}$ \\ Marlyn Romero Peñuela ${ }^{2}$ \\ Gonzalo Taborda Ocampo ${ }^{3}$
}

Recibido el 6 de agosto de 2013 y aprobado el 18 de febrero de 2014, actualizado el 9 junio de 2014

\section{RESUMEN}

El objetivo de este estudio fue determinar la presencia de residuos de oxitetraciclina (OTC) en 149 muestras de músculo diafragmático de bovinos de carne, en una planta de sacrificio comercial en Antioquia (Colombia). Las muestras fueron procesadas y purificadas mediante un sistema de extracción en fase sólida. La detección de OTC se realizó usando cromatografía líquida de alta eficiencia (HPLC), se usó como referencia el Límite Máximo de Residuos (LMR) de la Unión Europea (LMR > $100 \mathrm{ppb})$. El $49 \%$ de las muestras $(\mathrm{n}=73$ ) presentaron residuos de OTC; el $8 \%(n=12)$ de las muestras contenían cantidades de OCT superiores al LMR. La edad, la raza y la procedencia no estuvieron asociadas con la presencia de residuos de oxitetraciclina $(p>0,05)$. Las hembras presentaron una prevalencia más alta de residuos que los machos $(p=0,06)$. Se concluye que a pesar de que la prevalencia de residuos de OTC en la carne bovina evaluada fue baja, es evidente que se debe fortalecer la implementación de buenas prácticas ganaderas, un sistema de vigilancia epidemiológica y programas de entrenamiento para profesionales, ganaderos y personal responsable de los animales, a fin de garantizar un alimento inocuo al consumidor.

\section{PALABRAS CLAVE}

HPLC, tetraciclinas, inocuidad, ganado bovino, músculo.

\section{DETERMINATION OF OXYTETRACYCLINE RESIDUES IN SAMPLES OF BOVINE MEAT}

\begin{abstract}
The aim of this study was to determine the presence of oxytetracycline (OTC) residues in 149 samples of diaphragm muscle of beef cattle, in a commercial slaughterhouse in Antioquia (Colombia). The samples were processed and purified through solid phase extraction. OTC detection was performed using high performance liquid chromatography (HPLC), the Maximum Residue Limits (MRL) of the European Union was used as reference (MRL $>100 \mathrm{ppb}$ ). Forty-nine percent of the samples $(n=73)$ presented OTC residues, $8 \%(n=12)$ of samples contained amounts of OTC beyond the MRL. Age, breed and origin were not associated with the presence of oxytetracycline residues $(p>0.05)$. Prevalence of residues is higher among female than male $(p=0.06)$. It is concluded that although the prevalence of OTC residues in bovine meat evaluated was low, it is clear that the implementation of good farming practices, a system of epidemiological surveillance and training programs for professionals, livestock producers, and staff responsible for the animals should be strengthened, in order to guarantee safe food to consumers.
\end{abstract}




\section{KEY WORDS}

HPLC, tetracyclines, food safety, cattle, muscle.

\section{INTRODUCCIÓN}

Colombia es el cuarto productor de carne en América Latina, con un rebaño comercial de 27,7 millones de cabezas (FAOSTAT, 2010). Los avances en tecnologías incorporadas, menores costos de mano de obra y un gran mercado interno han fomentado el desarrollo de grandes operaciones en el procesamiento de la carne bovina (Estévez et al., 2009). El consumo per cápita de carne en el país en octubre del año 2012 fue 15,2 kg/habitante/año (SIPSA, Ministerio de Agricultura y Desarrollo Rural y DANE, 2012). Sin embargo, la carne es considerada como un alimento de alto riesgo epidemiológico, porque puede ser una vía importante de exposición a agentes bacterianos, al prión causante de la Encefalopatía Espongiforme Bovina (EEB), las dioxinas, los metales pesados, las hormonas y los residuos de medicamentos veterinarios (RMV) (Vásquez et al., 2002; Verbeke et al., 2007; Sofos, 2008). En especial los RMV y sus metabolitos son considerados un peligro químico para la salud pública y constituyen una preocupación para los consumidores, productores y organismos oficiales que velan por la inocuidad de los alimentos (Doyle, 2006).

Las tetraciclinas son quizás los antimicrobianos más ampliamente utilizados como promotores del crecimiento y en la terapéutica de animales de abasto, por su bajo costo, escasa toxicidad y amplio espectro, siendo uno de los medicamentos incluidos en los programas de vigilancia epidemiológica en todo el mundo (Oka et al., 1991). La Comunidad Europea ha reportado que más del $65 \%$ de los antibióticos prescritos para el uso terapéutico veterinario son tetraciclinas (2294 de 3494 ton) (Federación Europea de Sanidad Animal, 2001). En Colombia, la OTC es uno de los fármacos de mayor distribución, debido al número de registros (181) expedidos para su comercialización en el país, además de ser prescrito en todas las especies destinadas para consumo humano (Lozano y Arias, 2008). Los efectos adversos de la residualidad de este grupo de fármacos para los consumidores son: cambios en la flora intestinal e inhibiciones terapéuticas por el desarrollo de resistencia bacteriana (JECFA, 1990; Doyle, 2006; Lozano y Arias, 2008), riesgos teratogénicos, reacciones de hipersensibilidad (Reig y Toldrá, 2008) y manchas en los dientes de niños (Shahid et al., 2007).

A pesar de que Colombia cuenta con una política sanitaria y de inocuidad para las cadenas láctea y cárnica (Consejo Nacional de Política Económica y Social, 2005), con un reglamento técnico a través del cual se creó el Sistema Oficial de Inspección, Vigilancia y Control de la carne, productos cárnicos comestibles y derivados cárnicos (Ministerio de la Protección Social, 2007a) y se está implementando el Plan Nacional de Residuos de medicamentos veterinarios y contaminantes químicos para la carne, estos sistemas de control aún se encuentran en un estado incipiente de adopción, no son funcionales y los reportes oficiales sobre la situación de los medicamentos veterinarios en el país son insuficientes (Lozano y Arias, 2008; Márquez, 2008). El objetivo del presente estudio fue detectar y cuantificar los residuos de OCT en carne de ganado bovino sacrificado en una planta del noroccidente colombiano mediante la técnica de cromatografía líquida de alta eficiencia (HPLC) y establecer la asociación de los 
niveles residuales del fármaco con las variables edad, raza, género y procedencia de los animales.

\section{MATERIALES Y MÉTODOS}

El estudio se realizó durante los meses de abril a agosto de 2011, en una planta de sacrificio clase I apta para exportar, localizada en el departamento de Antioquia, la cual cumplía con los requerimientos sanitarios del Decreto 1500 de 2007 y la Resolución 2905 de 2007 (Ministerio de la Protección Social, 2007a, 2007b). La planta operaba de lunes a sábado (0600-1400 horas), con una capacidad de sacrificio de 500-600 bovinos/día (40-45 animales/hora).

\section{Descripción del estudio}

Se realizó un estudio de corte transversal en bovinos $(n=149)$, conformados por 135 machos y 14 hembras, que fueron manejados bajo condiciones comerciales, con características similares en cuanto a raza (cruces de ganado cebú Bos indicus y hembras de descarte Bos taurus), edad (20 a 60 meses) y peso vivo $(429,9 \pm 29,3 \mathrm{~kg})$. Los bovinos procedían de 69 fincas, localizadas en Córdoba y en las siguientes regiones del departamento Antioqueño: Valle de Aburrá, Magdalena Medio, Urabá, Bajo Cauca, y las zonas Norte, Suroeste y Occidente. Los bovinos fueron seleccionados aleatoriamente y se les realizó la inspección ante-mortem y post-mortem de conformidad con las directrices generales establecidas en el decreto 2905 del 2007, cuyos resultados se consignaron en el formato respectivo. De igual forma se registró la edad, raza, género y procedencia. Una vez fueron sacrificados, se recolectaron $500 \mathrm{~g}$ de músculo diafragmático, siguiendo el procedimiento establecido por el Codex Alimentarius (Codex Alimentarius, 2005, 2009), las cuales fueron almacenadas a $-20^{\circ} \mathrm{C}$ hasta el momento del análisis (Figura 1).

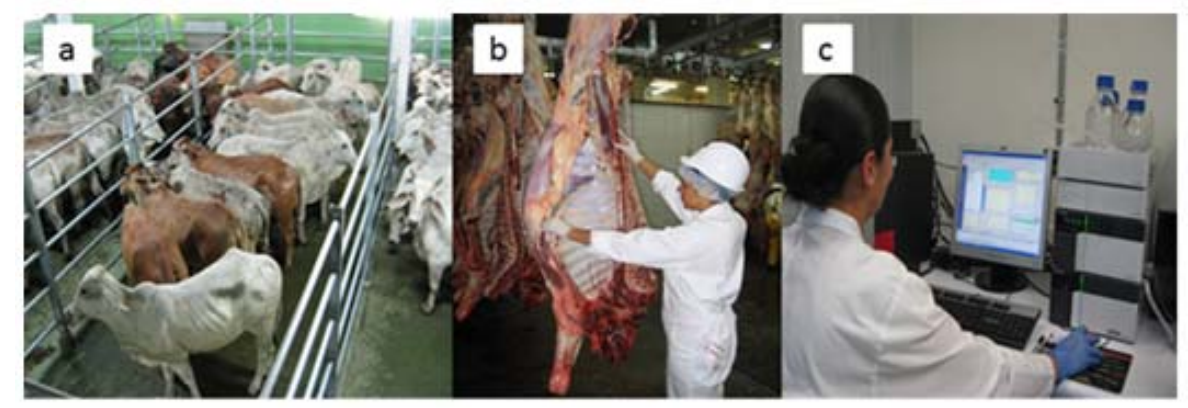

Figura 1. a) Bovinos evaluados. b) Toma de la muestras de músculo diafragmático. c) Análisis cromatográfico de las muestras.

\section{Preparación de la muestra}

La preparación de la muestra se realizó teniendo en cuenta los lineamientos descritos por la Asociación de Químicos Agrícolas Oficiales (AOAC, 1995). La muestra se descongeló a temperatura ambiente, se eliminó la grasa y se homogenizó. Se tomaron $5 \mathrm{~g}$ de la muestra y se colocaron en tubos de 
prolipropileno de $50 \mathrm{ml}$, se adicionaron $8 \mathrm{ml}$ de buffer Mcllvaine y se agitó durante 5 minutos. Se centrifugó a $3500 \mathrm{rpm}$ durante 10 minutos y el sobrenadante se transfirió a tubos nuevos, hasta alcanzar un volumen de $40 \mathrm{ml}$. El sobrenadante obtenido se filtró con membranas de microfibra de vidrio con un tamaño de poro de $55 \mathrm{~mm}$. Después se aisló el compuesto a través de un sistema de extracción en fase sólida (SPE), las columnas se acondicionaron con $20 \mathrm{ml}$ de metanol y $20 \mathrm{ml}$ de agua tipo I. Las muestras se hicieron pasar a través de las columnas, que posteriormente fueron lavadas con $20 \mathrm{ml}$ de agua. El analito fue eluido con $3 \mathrm{ml}$ de metanol, las muestras eluidas se secaron bajo una corriente suave de nitrógeno y se reconstituyeron en $1 \mathrm{ml}$ de fase móvil. Finalmente, las muestras fueron filtradas en membranas de nailon de $0,45 \mathrm{~mm}$ y depositadas en viales ámbar, para la separación y cuantificación en HPLC con detector UV-VIS (AOAC, 1995; Oka, Ito y Matsumoto, 2000; Cinquina et al., 2003; Cristofani et al., 2009).

\section{Análisis cromatográfico}

El nivel de OTC en las muestras extraídas se determinó usando cromatografía líquida de alta eficiencia (HPLC), según el método descrito por la AOAC (1995), con algunas modificaciones de la técnica realizadas por los autores. Los análisis se llevaron a cabo en un HPLC (Prominence LC-20AT, marca Shimadzu, Japón) y para la separación isocrática se empleó una columna C18 ODS2 (250 x 4,6 $\mathrm{mm}, 5 \mu \mathrm{m}$ ) marca Waters. La fase móvil estuvo compuesta de una solución acuosa de ácido oxálico 0,01 M: Acetonitrilo: Metanol (50:30:20, v/v/v) mantenida a un flujo de $1,5 \mathrm{ml} / \mathrm{min}$ a $40^{\circ} \mathrm{C}$. El analito se detectó mediante un detector de arreglo de diodos Prominence SPD-M20A marca Shimadzu operado a una longitud de onda de $360 \mathrm{~nm}$. El software empleado para el análisis de los datos obtenidos fue LC-Solution (Shimadzu). Los niveles de OTC se cuantificaron al medir las áreas de los picos y compararlas con los picos relevantes generados por el estándar, las muestras fueron inyectadas a razón de $50 \mu$ l.

A partir de la solución madre estándar se prepararon diferentes disoluciones en fresco disolviendo OTC en metanol, acetonitrilo y $0,01 \mathrm{M}$ de ácido oxálico (20:30:50). Se prepararon soluciones con concentración de 25, 100, 150, 200, y $300 \mu \mathrm{g} / \mathrm{L}$, las cuales fueron usadas para construir la curva de calibración. Todos los patrones fueron inyectados por triplicado y se obtuvo un promedio de las lecturas realizadas, la estimación de las cantidades de analito se realizó por interpolación de la gráfica obtenida. Una muestra se consideró positiva si su tiempo de retención y el pico correspondían con el estándar. Se estableció el punto de corte en 100 ppb para definir la presencia de OTC, que representa el límite máximo de residuos establecido (LMR) (Administración de Alimentos y Medicamentos de los Estados Unidos,1975; Commission Regulation 508/1999/EC, 1999; FAO/WHO, 2007).

\section{Análisis estadístico}

El análisis se efectúo utilizando el programa SAS (SAS, 2004). Mediante un análisis de varianza se evaluó el efecto de la raza, procedencia y el género sobre la prevalencia de muestras con residuos de OTC. Además, se realizó un análisis de regresión lineal simple para evaluar el efecto de la edad sobre la concentración de la OTC. Los resultados se reportaron como medias de mínimos cuadrados \pm error estándar (EE).

Para evaluar el efecto del género en la aparición de residuos de oxitetraciclina, se realizó un análisis del coeficiente de Pearson. La presencia de residuos se categorizó de la siguiente manera: muestras que excedían el LMR de la UE 
(concentración > $100 \mathrm{ppb}$ ) y muestras que no sobrepasaron el LMR (concentración $<100 \mathrm{ppb}$ ). Todos los valores de $\mathrm{p}<0,05$, fueron considerados como significativos.

\section{RESULTADOS}

La inspección ante-mortem y post-mortem estableció la aptitud sanitaria de los animales evaluados y no se realizaron decomisos. El $49 \%(73 / 149)$ de las muestras evaluadas presentó niveles detectables de OTC. De estas, el 16,43\% $(n=12)$ excedió el LMR definido por la UE en músculo, el $83,57 \%$ restante presentó niveles detectables del fármaco, pero sin exceder los límites aceptables. La media de los residuos de OTC en las 149 muestras fue de $73,1 \pm 38,9$ ppb y la cantidad máxima fue de 227,1 ppb.

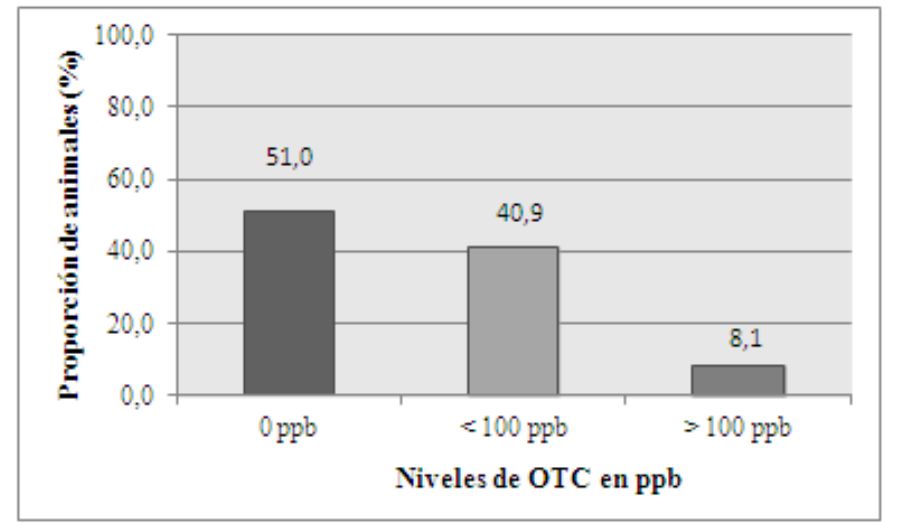

Figura 2. Niveles de OTC presentes en las muestras de músculo diafragmático ( $n=149$ animales).

No se presentaron diferencias significativas en los residuos de OTC, de acuerdo con la edad, raza, y procedencia $(p>0,05)$. Sin embargo, se presentó una tendencia con respecto al género de los animales $(p=0,06)$, dado que hubo mayor presencia de residuos de OTC en la hembras que en los machos (Tabla 1).

Tabla 1. Concentraciones de oxitetraciclina en carne bovina determinadas por HPLC

\begin{tabular}{|c|c|c|c|c|c|c|c|}
\hline \multirow[t]{2}{*}{ Género } & \multirow[t]{2}{*}{$\begin{array}{l}\text { Muestras } \\
\text { positivas/ } \\
\text { No. total }\end{array}$} & \multirow[t]{2}{*}{$\begin{array}{c}\% \text { de } \\
\text { muestras } \\
\text { positivas } \\
\text { a OTC }\end{array}$} & \multicolumn{3}{|c|}{$\begin{array}{l}\text { Concentración de OTC } \\
(\mathrm{ppb})^{1}\end{array}$} & $\begin{array}{l}\mathrm{LMR}^{2} \\
(\mathrm{ppb})^{1}\end{array}$ & \multirow{2}{*}{$\begin{array}{c}\text { No. de } \\
\text { muestras } \\
\text { sobrepasan } \\
\text { LMR } \\
\text { UE }^{3}\end{array}$} \\
\hline & & & Rango & Media & $\pm \mathrm{DE}$ & $\mathrm{UE}^{3}$ & \\
\hline Machos & $64 / 135$ & 47,4 & $28-227,1$ & 71 & 36,6 & 100 & 9 \\
\hline Hembras & $9 / 14$ & 64,3 & $37,6-192,6$ & 88.2 & 47,4 & 100 & 3 \\
\hline
\end{tabular}




\section{DISCUSIÓN}

En el presente estudio la prevalencia de residuos de OTC fue moderada, a diferencia de la reportada por otros investigadores en Colombia (Franco, Romero y Taborda, 2008), quienes mediante la técnica ELISA (Ensayo por inmunoabsorción ligado a enzimas) encontraron una alta proporción de muestras que excedieron los LMR establecidos por el Codex Alimentarius (CA) y la UE (61,5\% y el $23,7 \%$, respectivamente). Las diferencias en la prevalencia entre los dos estudios podrían estar relacionadas con la falta de implementación de las buenas prácticas ganaderas (BPG) en los predios de origen del ganado, el tipo de muestreo y la técnica empleada. En el presente estudio, la mayor proporción de los bovinos evaluados pertenecían a fincas certificadas en BPG; producto de una cuidadosa selección de los proveedores; el muestreo fue aleatorio e incluyó bovinos procedentes de 69 fincas, esto permitió tener una muestra más representativa y apreciar diferencias regionales en el uso de la OTC. A diferencia, en el estudio referido se seleccionaron 4 lotes que comprendían 29 animales de la misma procedencia (Franco et al., 2008). Respecto a las técnicas empleadas, los límites de detección (LD) de ambas son comparables, porque el LD fue de aproximadamente 2 ppb para ELISA y 2,39 ppb para HPLC. Estudios similares han referenciado prevalencias más altas. En Nairobi se obtuvieron prevalencias de OTC del 44\% ( $n=250$ muestras) (Muriuki et al., 2001), en Turkia el $70 \%$ de las muestras de carne curada analizada contenía residuos de OTC (Senyuva, Özden y Sarica, 2000) y en Bélgica el $86 \%$ de las muestras presentó LMR de tetraciclinas por encima de los niveles aceptables (Okerman, Van Hoof y Debeuckelaere, 1998).

Contrariamente a estos reportes, otros investigadores han presentado un panorama completamente diferente. Un estudio realizado en España encontró una prevalencia de $0,72 \%(4 / 550)$ para la presencia de residuos de tetraciclinas en carne, que superaron los LMR (Pérez de Ciriza et al., 1999). Por otro lado, Vásquez et al. (2002) en la región Noroeste de México, durante un período de 8 años analizaron 1034 muestras (músculo diafragmático, hígado, riñón y grasa) de las especies bovina, porcina y aviar, hallando una prevalencia de $2,5 \%$ de este mismo grupo de fármacos, sin exceder el LMR.

Los resultados discordantes de los niveles de residuos de tetraciclinas en muestras de carne en diferentes países, evidencian claras diferencias en cuanto al cumplimiento de los tiempos de retiro para este grupo de antibióticos en animales destinados al consumo, lo cual sugiere un uso indiscriminado (Muriuki et al., 2001) y la falta de implementación de buenas prácticas en la administración de medicamentos en las granjas, aspecto que ha sido reportado en Colombia (Romero y Sánchez, 2011). Esta situación se ve favorecida por las ventajas económicas que ciertos gobiernos ofrecen a los ganaderos (Abbasi et al., 2012), en el caso opuesto se ha visto cómo situaciones de austeridad en el campo, disminuyen el uso de estos productos en el sector pecuario (Gesche y Emilfork, 1998). Otros factores que promueven esta problemática son la ausencia de control en cuanto a la dosificación, frecuencia de aplicación y la falta de servicios de inspección oficial que vigilen la presencia de residuos y establezcan los LMR en productos de origen animal, como ocurre en algunos países (Alvarado, Ascanio y Méndez, 2008).

En la presente investigación se observó una alta proporción de muestras con niveles detectables de OTC $49 \%(73 / 149)$ y solo el $16 \%(n=12)$ de estas superaron los LMR. Resultados similares se obtuvieron en Ardabil provincia de Irán, donde hallaron una prevalencia de $9,1 \%$ en tejidos comestibles (músculo, riñón e hígado) de bovinos (Abbasi et al., 2012). En ambos casos se aprecia un 
amplio uso de estos fármacos con un cumplimiento parcial de los niveles permisibles. No obstante, los resultados no se pueden desestimar, porque sigue siendo un nivel importante de muestras contaminadas que pueden afectar la salud pública. Dentro de los aspectos que pudieron influir en la baja prevalencia de residuos en el actual estudio, sobresale la ausencia de hallazgos ante y postmortem, lo cual permite inferir que un estatus sanitario adecuado es un factor protector para la presentación de residuos en la carne (Codex Alimentarius, 2009; Gesche y Emilfork, 1998), teniendo en cuenta que es mucho mayor la persistencia de residuos en animales enfermos (Pérez de Ciriza et al., 1999). Otro aspecto a discutir es el tipo de muestra evaluada. Algunos investigadores consideran que en el músculo glúteo y el tríceps se pueden detectar mayores concentraciones de fármacos, al compararse con los hallados en el músculo diafragmático, que es la muestra recomendada por el CA para este tipo de análisis (Codex Alimentarius, 2005, 2009), debido a la proximidad de los puntos de inyección con el depósito del fármaco (Abbasi et al., 2012).

Un aspecto relevante en la investigación es la tendencia observada que identificó el género de los animales $(p=0,06)$ como un factor de riesgo, dado que hubo mayor presencia de residuos de OTC en las hembras que en los machos, al igual que en otros estudios (Alvarado et al., 2008). Esta tendencia puede estar relacionada con el uso indiscriminado de antibióticos sin cumplir con los tiempos de retiro, para el tratamiento de enfermedades como las afecciones reproductivas y la mastitis, en las hembras de descarte (Alvarado et al., 2008; Codex Alimentarius, 2009).

Se concluye que a pesar de que la prevalencia de residuos de OTC en la carne bovina evaluada fue baja, es evidente que se deben fortalecer las buenas prácticas de administración de medicamentos en la producción primaria, la implementación de programas de vigilancia epidemiológica y la capacitación de los ganaderos, profesionales y personal responsable de los animales, a fin de garantizar un producto inocuo al consumidor. De igual forma, se requiere el desarrollo de investigaciones que contemplen muestreos más representativos, con el fin de conocer la prevalencia real de residuos de medicamentos en alimentos de origen animal, como soporte al Plan Nacional de Residuos de Contaminantes en Alimentos bajo la coordinación del Instituto Nacional de Medicamentos y Alimentos (INVIMA) y el apoyo del Instituto Colombiano Agropecuario (ICA).

\section{AGRADECIMIENTOS}

A la Vicerrectoría de Investigaciones y Postgrados de la Universidad de Caldas por la financiación del proyecto, a los grupos de investigación CIENVET y GICTA por sus contribuciones, a Cristian Camilo Giraldo por el apoyo en el trabajo de campo, y al Dr. Henry Mesa Echeverri por la asesoría en el diseño experimental y en el análisis estadístico. 


\section{REFERENCIAS}

- Abbasi, M., Nemati, M., Babaei, H., Ansarin, M. y Nourdagar A (2012). Solid-phase extraction and simultaneous determination of tetracycline residues in edible cattle tissues using an HPLC-FL method. Iranian Journal of Pharmaceutical Research, 11(3), 781-787.

- Alvarado, S., Ascanio, E. y Méndez, C. (2008) Determinación de residuos de oxitetraciclina en muestras de tejido bovino destinadas al consumo humano. Rev. Fac. Cs. Vets, 49(2), 73-79.

- Association of Official Agricultural Chemist (AOAC). First Action (1995). Final Action (1999). Oficial method 995.09 Chortetracycline, Oxytetracicline and Tetracycline in edible animal tissues Liquid Chromatographic Method.

- Cinquina, A., Longo, F., Anastasi, G., Giannetti, L. y Cozzani, R. (2003) Validation of a high-performance liquid chromatography method for the determination of oxytetracycline, tetracycline, chlortetracycline and doxycycline in bovine milk and muscle. J. Chromatogr. B., 997, 227-233.

- Codex Alimentarius. (2005). Límites máximos de residuos para medicamentos veterinarios en los alimentos. CAC/LMR 02. 1-25.

- . (2009). Directrices para el diseño y la implementación de programas nacionales reglamentarios de aseguramiento de inocuidad alimentaria relacionados con el uso de medicamentos veterinarios en los animales destinados a la producción de alimentos. CAC/GL 71. 1-33.

- Commission Regulation 508/1999/EC (1999). Amending Annexes I-IV to council Regulation (EEC) No 2377/90 laying down Community procedure for the establishment of maximum residue limits of veterinary medicinal products in foodstuffs of animal origin. Official Journal of European Communities, L224, 16. Recuperado de http://europa.eu.int

- Consejo Nacional de Política Económica y Social. (2005). Documento Conpes 3376. Política sanitaria y de inocuidad para las cadenas de la carne bovina y de la leche. Bogotá, Colombia.

- Cristofani,E., Antonini,C., Tovo, G., Fioroni, L., Piersanti, A. y Galarini, R. (2009). A confirmatory method for the determination of tetracyclines in muscle using high-performance liquid chromatography with diode-array detection. Analytica Chimica Acta, 637, 40-46.

- Doyle, M. (2006). Veterinary drug residues in processed meats Potential health risk. A review of the Scientific Literature. Food Research Institute, University of Wisconsin-Madison. Wisconsin, United States.

- Estévez, L., Santana, A., Camacho, C., Gutiérrez, J., Gómez, M., García, G., Rozo, M. y Ballesteros, H. (2009). Competir e innovar: la ruta de la industria bovina. Agenda prospectiva de investigación y desarrollo tecnológico para la cadena cárnica bovina en Colombia. Bogotá, Colombia: Ministerio de Agricultura y Desarrollo Rural, FEDEGAN, CORPOICA, Universidad Nacional de Colombia.

- European Federation of Animal Health (FEDESA). (2001). Antibiotic Use in Farm Animals does not threaten Human Health. FEDESA/FEFANA. Brussels, Belgium.

- FAOSTAT (2010). Online database of the Food and Agriculture Organization of the United Nations. Recuperado de http://faostat.fao.org/site/573/DesktopDefaul.aspx?PagelD=573\#ancor

- FAO/WHO Food Standards Codex Alimentarius. (2007). Veterinary drug residues in food. Maximum residues limits. Recuperado de http://www.codexalimentarius.net/mrls/vetdrugs/jsp/vetd_q-e.jsp

- Franco, J., Romero, M. y Taborda, G. (2008). Determinación de niveles residuales de tetraciclina en canales bovinas por la técnica de ELISA en el frigorífico FRIOGAN (La Dorada). Biosalud, 7, 47-55. 
- Gesche, E. y Emilfork, C. (1998). Residuos de antimicrobianos en canales de vacas. Arch. Med. Vet., 30(2), 137-143.

- JECFA Joint FAO/WHO. (1990). Expert Committee on Food Additives. Evaluation of certain veterinary drug residues in food. WHO Technical Report Series No. 799, Geneve, Italy.

- Lozano, M. y Arias, D. (2008). Residuos de fármacos en alimentos de origen animal: panorama actual en Colombia. Rev Colomb Cienc Pecu., 21, 121-135.

- Márquez, L. (2008). Residuos químicos en alimentos de origen animal: problemas y desafíos para la inocuidad alimentaria en Colombia. Revista Corpoica - Ciencia y Tecnología Agropecuaria, 9(1), 124-135.

- Ministerio de la Protección Social. (2007a). Decreto 1500. Bogotá, Colombia.

- . (2007b). Resolución 2905. Bogotá, Colombia.

- Muriuki, F., Ogara, W., Njeruh, F. y Mitema, E. (2001). Tetracycline residue levels in cattle meat from Nairobi slaughter house in Kenya. J. Vet. Sci., 2(2), 97-101.

- Oka, H., Ikai, Y., Kawamura, N. y Hayakawa, J. (1991). Limited survey of residual tetracyclines in tissues collected from diseased animals in Aichi prefecture, Japan. J. Assoc. Off. Anal. Chem., 74, 894-896.

- Oka, H., Ito, Y. y Matsumoto, H. (2000). Chromatographic analysis of tetracycline antibiotics in foods. J. Chromatogr. A., 882, 109-133.

- Okerman, L., Van Hoof, J. y Debeuckelaere, W. (1998). Evaluation of the European four-plate test as a tool for screening antibiotic residues in meat samples from retail outlets. J. AOAC Int., 81, 51-56.

- Pérez de Ciriza, J., Huarte, A., Saiz, I., Ozcáriz, M. y Purroy. (1999). M. Residuos de sustancias inhibidoras en carnes. Anales Sis. San Navarra, 22(3), 231-238.

- Reig, M. y Toldrá, F. (2008). Veterinary drug residues in meat: concerns and rapid methods for detection. Meat Science, 78(1,2), 60-67.

- Romero, M. y Sánchez, J. (2011). Evaluación de las buenas prácticas ganaderas en bovinos de carne en el centro de Caldas. Biosalud, 10(1), 52-60.

- SAS Institute Inc. (2004). SAS/STAT® 9.1 User's Guide. Cary, NC: SAS Institute Inc.

- Senyuva, H., Özden, T. y Sarica, D. (2000). High-performance liquid chromatographic determination of oxytetracycline residue in cured meat products. Turk J Chem, 24, 395-400.

- Shahid, M., Siddique, M., Abubakar, M., Arshed, M., Asif, M. y Ahmad A. (2007). Status of Oxytetracycline residues in chicken meat in Rawalpindi/Islamabad Area of Pakistan. Asian Journal of Poultry Science, 1(1), 8-15.

- Sistema de Información de Precios y Abastecimiento del Sector Agropecuario (SIPSA), Ministerio de Agricultura y Desarrollo Rural y Departamento Administrativo Nacional de Estadística (DANE). (2012). La estructura de la producción de carne bovina en Colombia. Boletín mensual, Insumos y factores asociados a la producción agropecuaria, 4, 17.

- Sofos J. (2008). Challenges to meat safety in the 21St century. Meat Science, 78, 3-13.

- US Food and Drug Administration (1975). Titled 21-Food and drugs. Chapter 1 - Food and Drugs Administration Department of Health and Human Services, Subchapter E - Animal drugs - feed and related products. Part 556. Tolerances for residues of new animal drugs in food. Recuperado 
http://www.accessdata.fda.gov/scripts/cdrh/cfdocs/cfcfr/CFRSearch.0 063fm?CFRPart $=556$

- Vásquez, L., Bermúdez, M., García, L., Languré, A., Flores, M. y Orantes, C. (2002). Estudio de residuos tóxicos en tejidos animales destinados al consumo. Revista Científica, 12(3), 186-192.

- Verbeke, W., Frewer, L., Scholderer, J. y De Brabander, H. (2007). Why consumers behave as they do with respect to food safety and risk information. Anal Chim Acta, 586, 2-7.

1. MVZ, M.Sc. (c). Grupo de Investigación CIENVET, Facultad de Ciencias Agropecuarias, Universidad de Caldas, Manizales, Colombia. sandramil9@hotmail.com

2. MVZ, Esp., M.Sc., Ph.D. Profesora Departamento de Salud Animal, Grupo de Investigación CIENVET, Facultad de Ciencias Agropecuarias, Universidad de Caldas, Manizales, Colombia. marlyn.romero@ucaldas.edu.co

3. Lic. Qui., M.Sc., Ph.D. Profesor Departamento de Química, Grupo de Investigación GICTA, Facultad de Ciencias Exactas, Universidad de Caldas, Manizales, Colombia. gonzalo.taborda@ucaldas.edu.co

Para citar este artículo: Acosta-Agudelo, S.M., Romero-Peñuela, M., TabordaOcampo, G. (2014). Determinación de residuos de oxitetraciclina en muestras de carne bovina. Revista Luna Azul, 39, 143-152. Recuperado de http://lunazul.ucaldas.edu.co/index.php?option=content\&task=view\&id=952 